\title{
Roles of Critical Valence Fluctuations in Ce- and Yb-Based Heavy Fermion Metals
}

\author{
Shinji Watanabe, Kazumasa Miyake \\ Graduate School of Engineering Science, Osaka University, Toyonaka, Osaka 560-8531, Japan
}

\begin{abstract}
The roles of critical valence fluctuations of $\mathrm{Ce}$ and $\mathrm{Yb}$ are discussed as a key origin of several anomalies observed in Ce- and Yb-based heavy fermion systems. Recent development of the theory has revealed that a magnetic field is an efficient control parameter to induce the critical end point of the first-order valence transition. Metamagnetism and non-Fermi liquid behavior caused by this mechanism are discussed by comparing favorably with $\mathrm{CeIrIn}_{5}$, $\mathrm{YbAgCu}_{4}$, and $\mathrm{YbIr}_{2} \mathrm{Zn}_{20}$. The interplay of the magnetic order and valence fluctuations offers a key concept for understanding Ce- and Yb-based systems. It is shown that suppression of the magnetic order by enhanced valence fluctuations gives rise to the coincidence of the magnetictransition point and valence-crossover point at absolute zero as a function of pressure or magnetic field. The interplay is shown to resolve the outstanding puzzle in $\mathrm{CeRhIn}_{5}$ in a unified way. The broader applicability of this newly clarified mechanism is discussed by surveying promising materials such as $\mathrm{YbAuCu}_{4}, \beta-\mathrm{YbAlB}_{4}$, and $\mathrm{YbRh}_{2} \mathrm{Si}_{2}$.
\end{abstract}

\section{Introduction}

In this article, we discuss the roles of critical valence fluctuations of $\mathrm{Ce}$ and $\mathrm{Yb}$ as a key origin of several anomalies observed in the Ce- and Yb-based heavy fermion systems. Recent development of the theory and experiments have revealed that the valence fluctuations appear ubiquitously by tuning the control parameters of the magnetic field and pressure, giving rise to broader consequences than previously recognized. First, we briefly survey accumulated experiments on the anomalies related to the valence transition and its fluctuations, and explain how the theoretical understanding has been achieved so far. Then, we discuss the recent development of theory and experimental results focusing on the prototypical materials.

Valence transition is an isostructural phase transition with a valence of the materials element showing a discontinuous jump. A typical example is known as the $\gamma-\alpha$ transition in Ce metal [1], where the first-order valence transition (FOVT) occurs in the temperature-pressure $(T, P)$ phase diagram while keeping the fcc lattice structure, as shown in Fig. 1(a). The critical end point (CEP) of the FOVT is located at $(T, P)=(600 \mathrm{~K}, 2 \mathrm{GPa})$. The valence of Ce changes discontinuously between $\mathrm{Ce}^{+3.03}\left(\gamma\right.$ phase) and $\mathrm{Ce}^{+3.14}(\alpha$ phase) at $T=300 \mathrm{~K}[2]$. YbInCu is also well known as a prototypical material for the isostructural FOVT [3, 4], where as $T$ decreases, the $\mathrm{Yb}$ valence changes discontinuously from $\mathrm{Yb}^{+2.97}$ to $\mathrm{Yb}^{+2.84}$ at $T=42 \mathrm{~K}[5]$. As diverging density fluctuations in the liquid-gas transition, valence fluctuations diverge at the CEP of the FOVT. When the temperature of the CEP is suppressed by controlling the materials parameters and enters the Fermi degeneracy regime, diverging valence fluctuations are considered to be coupled to the Fermi-surface instability. This multiple instability is considered 
to be a key mechanism for understanding anomalies observed in Ce- and Yb-based heavyfermion systems. Such phenomena have been detected in $\mathrm{CeCu}_{2} \mathrm{Ge}_{2}$ [6] and $\mathrm{CeCu}_{2} \mathrm{Si}_{2}$ [7], where remarkable enhancement of the superconducting transition temperature appears at the pressure $P_{\mathrm{v}}$ larger than $P_{\mathrm{c}}$ corresponding to the antiferromagnetic $(\mathrm{AF})$ quantum critical point $(\mathrm{QCP})$ (see Fig. 1(b)), and in $\mathrm{CeCu}_{2}\left(\mathrm{Si}_{x} \mathrm{Ge}_{1-x}\right)_{2}$ where two separate domes of the superconducting phase have been found under pressure [8]. In these materials, near $P=P_{\mathrm{v}}$, remarkable anomalies such as the $T$-linear resistivity and enhancement of the residual resistivity have been observed, which clearly exhibit distinct behavior of resistivity near $P=P_{\mathrm{c}}[6,7,8]$. Furthermore, these materials show that the coefficient $A$ obtained by the $T^{2}$ fitting to the lowest- $T$ regime of the resistivity decreases by 2-3 orders of magnitude when $P$ increases across $P=P_{\mathrm{v}}$.

Theoretically, it has been pointed out that enhanced Ce-valence fluctuations are the possible origin of the anomalies near $P=P_{\mathrm{v}}$ in the $\mathrm{CeCu}_{2}(\mathrm{Si} / \mathrm{Ge})_{2}$ systems [9]. It has also been pointed out that the sudden drop of the coefficient $A$ at $P=P_{\mathrm{v}}$ can be understood as a sharp change of the valence of $\mathrm{Ce}$ on the basis of the Gutzwiller arguments in the periodic Anderson model [10]. It has been also shown that enhanced valence fluctuations cause the $T$-linear resistivity 7 and enhancement of residual resistivity [11. The emergence of the superconducting dome near $P \sim P_{\mathrm{v}}$ in Fig. 1(b) has been explained by the valence-fluctuation-mediated superconductivity shown by the slave-boson mean-field theory taking account of the Gaussian fluctuations in the periodic Anderson model [10]. The density-matrix renormalization group (DMRG) calculation applied to the same model in one spatial dimension has also shown the enhancement of the superconducting correlation in the same parameter regime [12. Pairing symmetry of densityfluctuation (i.e., valence-fluctuation)-mediated superconductivity has also been analyzed on the basis of the phenomenological model [13].

Furthermore, recent NQR measurement in $\mathrm{CeCu}_{2} \mathrm{Si}_{2}$ has detected change of the $\mathrm{Cu}-\mathrm{NQR}$ frequency near $P=P_{\mathrm{v}}$, suggesting that the Ce valence changes near $P=P_{\mathrm{v}}$ [14]. Detailed measurement of the $T$ dependence of the specific heat and the upper critical field in $\mathrm{CeCu}_{2} \mathrm{Si}_{2}$ under pressure concluded that the pairing symmetries of the superconducting phases near $P=P_{\mathrm{c}}$ and $P=P_{\mathrm{v}}$ are different, suggesting that a pairing mechanism different from the antiferromagnetic spin-fluctuation mediated one is realized near $P_{\mathrm{v}}$ [15]. For a detailed summary of the theory and related experiments up to this stage, readers can refer to Ref. [16] and also Ref. [17].

Recent development of the theory of the quantum critical end point (QCEP) of the FOVT has revealed that valence fluctuations play a key role in the other Ce- and Yb-based heavy fermion systems more ubiquitously. We discuss the crucial roles of valence fluctuations in the following sections. In $\S 2$, we discuss the properties of the valence transition and valence crossover of Ce or $\mathrm{Yb}$ by comparing theoretical phase diagrams with Ce- and Yb-based systems. In $\S 3$, we discuss the fact that the QCEP of the FOVT can be induced rather easily by applying the magnetic field to the Ce- and Yb-based systems, which causes various anomalies such as metamagnetism and non-Fermi liquid behavior. We discuss that this mechanism gives a unified explanation for $\mathrm{CeIrIn}_{5}, \mathrm{YbAgCu}_{4}$, and $\mathrm{YbIr}_{2} \mathrm{Zn}_{20}$. In $\S 4$, we discuss the interplay of magnetic order and valence fluctuations, giving rise to coincidence of the magnetic transition and sharp valence crossover at $T=0 \mathrm{~K}$, as shown in Fig. 1(c). We show how the interplay resolves the outstanding puzzle in $\mathrm{CeRhIn}_{5}$ and also in $\mathrm{YbAuCu}_{4}$. The summary and outlook are given in $\S 5$. The broader applicability of the present mechanism is discussed by surveying promising materials such as $\beta-\mathrm{YbAlB}_{4}$ and $\mathrm{YbRh}_{2} \mathrm{Si}_{2}$. 

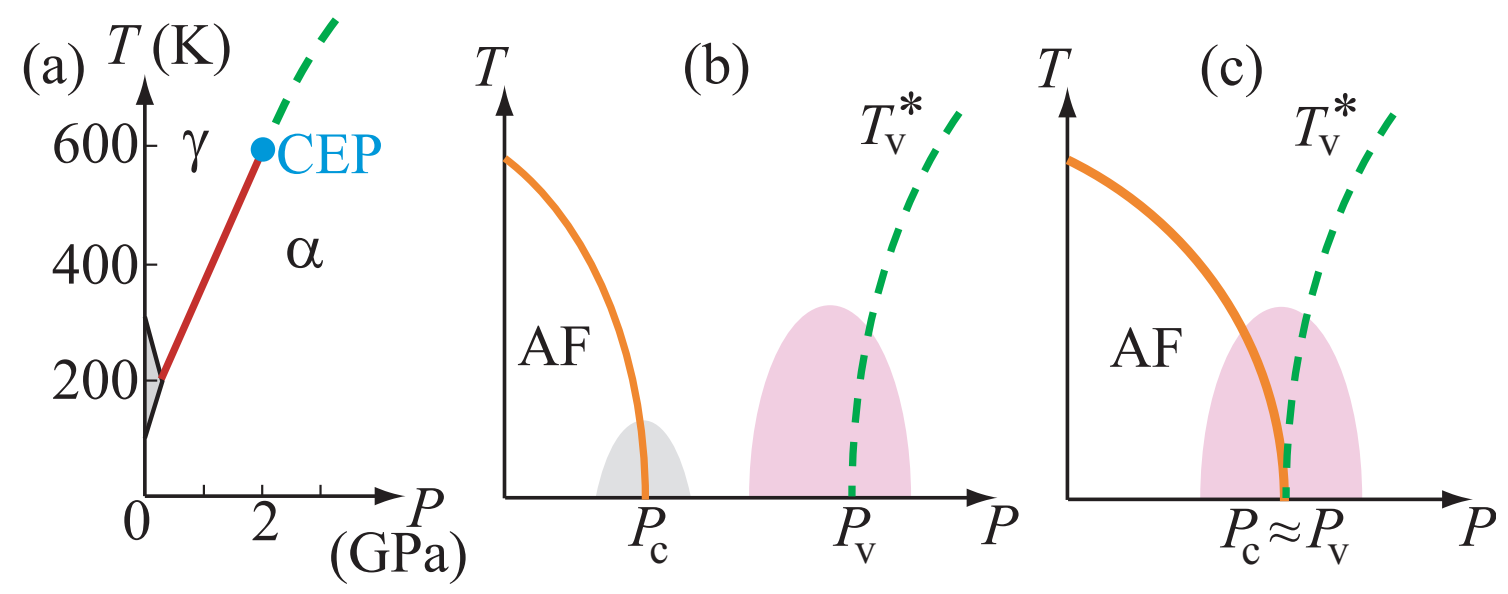

Figure 1. (color online) (a) $T$-P phase diagram of Ce metal. The first-order valence transition line (bold line) terminates at a critical end point (filled circle). Schematic $T$ - $P$ phase diagrams for (b) $P_{\mathrm{c}}<P_{\mathrm{v}}$, and (c) $P_{\mathrm{c}} \approx P_{\mathrm{v}}$. The dashed line represents valence-crossover temperature $T_{\mathrm{v}}^{*}$ (see text). In (b) and (c), shaded regions represent superconducting phases.

\section{Model and phase diagrams}

2.1. Minimal model for Ce- and Yb-based heavy fermions exhibiting valence transition

We consider the simplest minimal model, which describes an essential part of the physics in Ceand Yb-based heavy fermion systems exhibiting valence transition, as follows:

$$
\mathcal{H}=H_{\mathrm{c}}+H_{\mathrm{f}}+H_{\mathrm{hyb}}+H_{U_{\mathrm{fc}}}
$$

where $H_{\mathrm{c}}=\sum_{\mathbf{k} \sigma} \varepsilon_{\mathbf{k}} c_{\mathbf{k} \sigma}^{\dagger} c_{\mathbf{k} \sigma}$ represents the conduction band, $H_{\mathrm{f}}=\varepsilon_{\mathrm{f}} \sum_{i \sigma} n_{i \sigma}^{\mathrm{f}}+U \sum_{i=1}^{N} n_{i \uparrow}^{\mathrm{f}} n_{i \downarrow}^{\mathrm{f}}$ the $\mathrm{f}$ level $\varepsilon_{\mathrm{f}}$ and onsite Coulomb repulsion $U$ for $\mathrm{f}$ electrons, $H_{\mathrm{hyb}}=V \sum_{i \sigma}\left(f_{i \sigma}^{\dagger} c_{i \sigma}+c_{i \sigma}^{\dagger} f_{i \sigma}\right)$ the hybridization $V$ between $\mathrm{f}$ and conduction electrons, and $H_{U_{\mathrm{fc}}}=U_{\mathrm{fc}} \sum_{i=1}^{N} n_{i}^{\mathrm{f}} n_{i}^{c}$ the Coulomb repulsion $U_{\mathrm{fc}}$ between $\mathrm{f}$ and conduction electrons. The $H_{U_{\mathrm{fc}}}$ term is a key ingredient for explaining the valence transition as well as the various anomalies caused by enhanced Ce- or Yb-valence fluctuations [10, 12, 16, 18, 17): The T-linear resistivity and residual resistivity peak have also been shown theoretically by the model Eq. (1) [7, 11.

\subsection{Ground state and finite-T phase diagrams}

Figure 2(a) shows the schematic ground-state phase diagram for paramagnetic states drawn on the basis of the DMRG calculation in one-spatial dimension $(d=1)[12$ and the calculation by the dynamical-mean-field-theory (DMFT) in infinite-spatial dimension $(d=\infty)$ [19] applied to the model Eq. (1). Here, the filling $n \equiv\left(n_{\mathrm{f}}+n_{\mathrm{c}}\right) / 2$ with $n_{\mathrm{a}} \equiv \sum_{\sigma} \sum_{i=1}^{N}\left\langle a_{i \sigma}^{\dagger} a_{i \sigma}\right\rangle / N$ for a=f or c with $N$ being the number of lattice sites is set slightly smaller than 1 (half filling), which describes typical Ce- and Yb-based heavy-fermion metals.

The FOVT line (solid line) terminates at the QCEP (filled circle) and sharp valence crossover occurs (dashed line). When $\varepsilon_{\mathrm{f}}$ is deep enough, the Kondo state with $n_{\mathrm{f}}=1$ is realized. As $\varepsilon_{\mathrm{f}}$ increases across the FOVT and valence-crossover line, the mixed valence (MV) state with $n_{\mathrm{f}}<1$ is realized [20]. At the QCEP, the valence fluctuation $\chi_{\mathrm{v}} \equiv-\partial n_{\mathrm{f}} / \partial \varepsilon_{\mathrm{f}}$ diverges and at the valence-crossover line, enhanced valence fluctuation appears [12, 18. We note that the slave-boson mean-field theory for $d=1[12, d=2[21$, and $d=3$ [22, 18 also gives essentially the same phase diagram as Fig. 2(a). This is because the valence transition and its fluctuation 

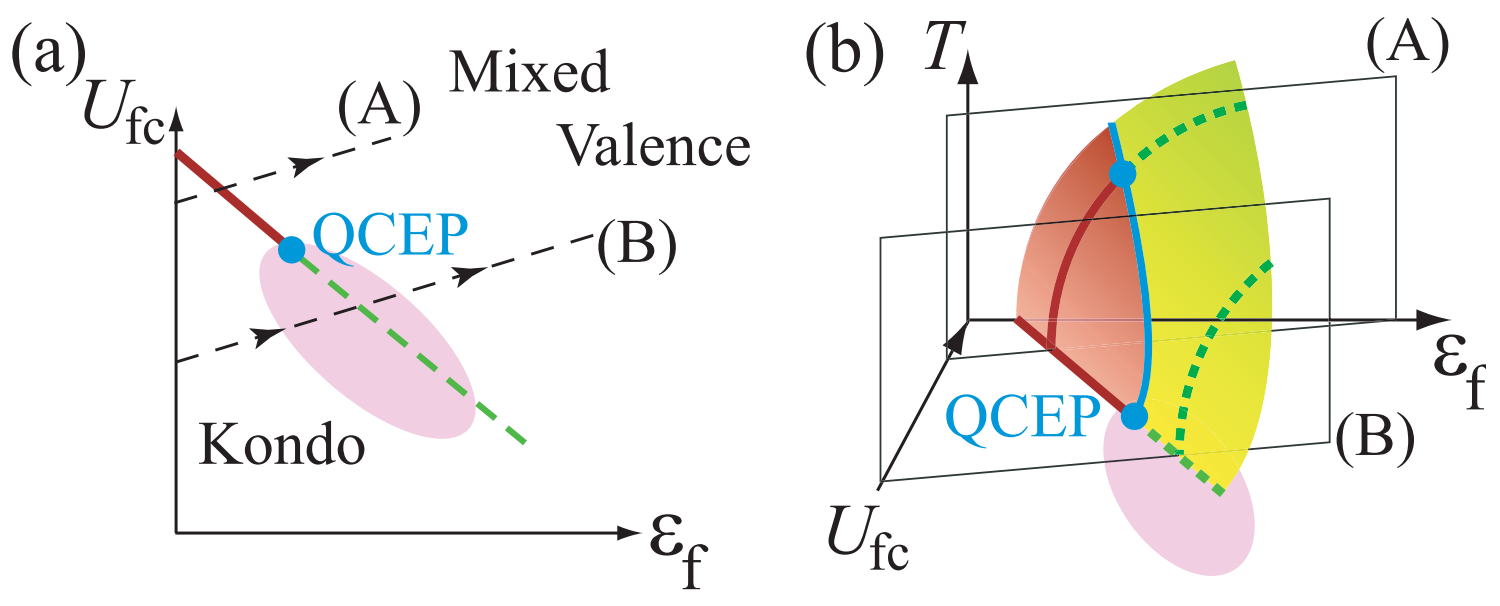

Figure 2. (color online) Schematic phase diagrams of (a) $\varepsilon_{\mathrm{f}}-U_{\mathrm{fc}}$ plane at $T=0 \mathrm{~K}$ and (b) $T-\varepsilon_{\mathrm{f}}-U_{\mathrm{fc}}$ space for finite c-f hybridization $V$ and large $U$ in the periodic Anderson model Eq. (1D) (see text). The first order valence transition line (bold line) terminates at the quantum critical end point (QCEP) (filled circle) and sharp valence crossover occurs on the thick dashed line. In (b), the FOVT surface (dark surface) and the valence-crossover surface (light surface) are separated by the critical-end line (thick line) touched at $T=0 \mathrm{~K}$, which is the QCEP. In (a) and (b), shaded regions at $T=0 \mathrm{~K}$ represent superconducting phases. The thin dashed lines with arrow(s) in (a) represent routes for applying pressure to Ce metal (A) and Ce-based compounds (B), respectively. Corresponding $T$ - $P$ phase diagrams are shown by the cutout (A) and (B) in (b), which correspond to Fig. 1(a) and Fig. 1(b) (or Fig. 1(c)), respectively (see text).

are caused by the atomic Coulomb interaction $U_{\mathrm{fc}}$ in Eq. (1), which is ascribed to the local origin. Hence, the essential feature of the phase diagram for the valence transition does not depend on the spatial dimension. As shown by the shaded region in Fig. 2(a), near the QCEP of the FOVT, the superconducting phase is shown to appear by the slave-boson mean field theory taking into account the Gaussian fluctuations [10], which is supported by the DMRG calculation in the same model Eq. (11) in $d=1$ [12].

Here, we remark that the valence transition is completely different from the localized-toitinerant transition of $f$ electrons. Everywhere in the phase diagram in Fig. 2(a), f electrons are itinerant with finite c-f hybridization $\left\langle f_{\mathbf{k} \sigma}^{\dagger} c_{\mathbf{k} \sigma}\right\rangle \neq 0$. Hence, the number of $\mathrm{f}$ electrons is always included in the total volume of the Fermi surface, giving rise to the large Fermi surface (see also Fig. 6(b)). This is naturally understood in terms of the Landau-Luttinger sum rule. The existence of the QCEP in the ground-state phase diagram ensures that adiabatic continuation holds between the Kondo state and the MV state by detouring the QCEP of the first-ordertransition line.

Figure 2(b) shows the schematic phase diagram in the $T-\varepsilon_{\mathrm{f}}-U_{\mathrm{fc}}$ space. The FOVT surface (dark surface) bends to the side of the MV regime for at least large $U_{\mathrm{fc}}$ since larger entropy coming from f-spin degrees of freedom in the Kondo regime earns the larger free-energy gain [18]. The FOVT line in Fig. 2(a) is the line at the bottom of the FOVT surface. The critical-end line exhibiting diverging valence fluctuations, i.e., $\chi_{\mathrm{v}}=\infty$, separates the FOVT surface and the valence-crossover surface (light surface), which touches the $T=0$ plane at the QCEP. We note that even at the valence-crossover surface, valence fluctuations are enhanced [18, which cause remarkable anomalies in physical quantities in Ce- and Yb-based materials, as will be shown in $\S 3$ and $\S 4$. 


\subsection{Correspondence to $\mathrm{Ce}$ - and $\mathrm{Yb}$-based materials}

As shown in Fig. 2(a), the Kondo (MV) state with $n_{\mathrm{f}} \approx 1\left(n_{\mathrm{f}}<1\right)$ is realized for deep (shallow) $\varepsilon_{\mathrm{f}}$ for a fixed $U_{\mathrm{fc}}$. In Ce systems, the $n_{\mathrm{f}}=1$ state corresponds to $\mathrm{Ce}^{+3.0}$ with a $4 \mathrm{f}^{1}$-electron configuration per Ce site. In $\mathrm{Yb}$ systems, where $\mathrm{Yb}^{+3.0}$ has $4 \mathrm{f}^{13}$-electron configuration per $\mathrm{Yb}$ site, the hole picture is useful, so that the $n_{\mathrm{f}}=1$ state corresponds to $\mathrm{Yb}^{+3.0}$ with a $4 \mathrm{f}^{1}$-hole configuration per $\mathrm{Yb}$ site.

When we apply pressure to the $\mathrm{Ce}(\mathrm{Yb})$ compounds, $\varepsilon_{\mathrm{f}}$ increases (decreases), since negative ions approach the tail of the wavefunction of $4 \mathrm{f}$ electrons (holes) at the $\mathrm{Ce}(\mathrm{Yb})$ site. The hybridization $V$ and inter-orbital Coulomb interaction $U_{\mathrm{fc}}$ also increase. In the case of Ce metal, 4f and $5 \mathrm{~d}$ electrons are located at the same Ce site, which makes $U_{\mathrm{fc}}$ large [1. In Fig. 2(a), the dashed line with an arrow (A) represents the route for applying the pressure to Ce metal. Hence, the cutout for the large $U_{\mathrm{fc}}$ including the $T$ axis labeled by (A) in Fig. 2(b) corresponds to the $T$ - $P$ phase diagram of Ce metal shown in Fig. 1(a). Namely, the large critical-end temperature $T \sim 600 \mathrm{~K}$ in Ce metal is naturally understood from a large $U_{\mathrm{fc}}$ due to its on-site origin in Fig. 2(b).

On the other hand, in the case of $\mathrm{Ce}(\mathrm{Yb})$ compounds with conduction electrons supplied from elements other than $\mathrm{Ce}(\mathrm{Yb})$, the Coulomb repulsion between the $4 \mathrm{f}$ electron at the $\mathrm{Ce}$ $(\mathrm{Yb})$ site and the conduction electron $U_{\mathrm{fc}}$ is weaker in general because of its inter-site origin. Hence, applying pressure to the Ce compounds corresponds to the route represented by the dashed line with arrows (B) in Fig. 2(a). The cutout for such a moderate $U_{\text {fc }}$ labeled by (B) in Fig. 2(b) is considered to correspond to the $T$ - $P$ phase diagrams of most of the Ce-based compounds. Although the magnetically-ordered phase is not shown in Figs. 2(a) and 2(b) for simplicity of explanation here, the cutout (B) corresponds to Fig. 1(b) (or Fig. 1(c)). Namely, in $\mathrm{CeCu}_{2}(\mathrm{Si} / \mathrm{Ge})_{2}$ systems, the AF phase is located in the Kondo regime with a certain interval to the valence-crossover pressure $P_{\mathrm{v}}$ in Fig. 1(b), where the valence-crossover temperature $T_{\mathrm{v}}^{*}(P)$ covered by the superconducting dome corresponds to the cutout of Fig 2(b). When we take into account the magnetic order in the phase diagram, depending on the strength of the c-f hybridization $V$ (and also $\varepsilon_{\mathrm{f}}$ and $U_{\mathrm{fc}}$ ) in Eq (11), the location of the magnetic-paramagnetic phase boundary changes. Hence, the relative position of $P_{\mathrm{c}}$ and $P_{\mathrm{v}}$ changes as shown in Figs. 1(b) and 1(c). The interplay of the magnetic order and valence crossover (or transition) is quite important in understanding the actual phase diagrams of Ce- and Yb-based systems, which will be discussed in $\S 4$.

Here, we remind the readers of the fact that even at the $\gamma-\alpha$ transition in Ce metal, the magnitude of the valence jump is about 0.10 [2] as it is in the case of $\mathrm{YbInCu}_{4}$ [5], as remarked in $\S 1$. We note that the magnitude of the valence change at the valence crossover for moderate $U_{\mathrm{fc}}$ is smaller than that at the FOVT for large $U_{\mathrm{fc}}$, which is expected to be in the order of 0.01 . Actually, such a tiny change of the $\mathrm{Yb}$ valence has been observed at the valence-crossover temperature $T=T_{\mathrm{v}}^{*} \sim 40 \mathrm{~K}$ in $\mathrm{YbAgCu}_{4}$ [35], which will be discussed in $\S 3.3$.

\section{Field-induced valence crossover}

\subsection{Theoretical results}

As shown in $\S 2$, the valence transition is essentially ascribed to the charge degrees of freedom and it is nontrivial how the magnetic field affects the valence transition. Recently, theoretical studies have clarified that the QCEP of the FOVT as well as the sharp valence crossover is induced by applying a magnetic field [18. The slave-boson mean-field theory applied to the model Eq. (11) in $d=3$ with the Zeeman term $-h \sum_{i}\left(S_{i}^{\mathrm{f} z}+S_{i}^{\mathrm{c} z}\right)\left(h \equiv g \mu_{\mathrm{B}} H\right)$ has shown that the FOVT surface and the valence-crossover surface move as shown in Fig. 3(a) [22, 18]. On the locus with an arrow illustrated in Fig. 3(a), the magnetic susceptibility $\chi=\partial m / \partial h$ with

$m \equiv \sum_{i}\left\langle f_{i \uparrow}^{\dagger} f_{i \uparrow}-f_{i \downarrow}^{\dagger} f_{i \downarrow}+c_{i \uparrow}^{\dagger} c_{i \uparrow}-c_{i \downarrow}^{\dagger} c_{i \downarrow}\right\rangle / N$ diverges. Namely, metamagnetism occurs. The DMRG calculation applied to the same model in $d=1$ has also shown that the field-induced 

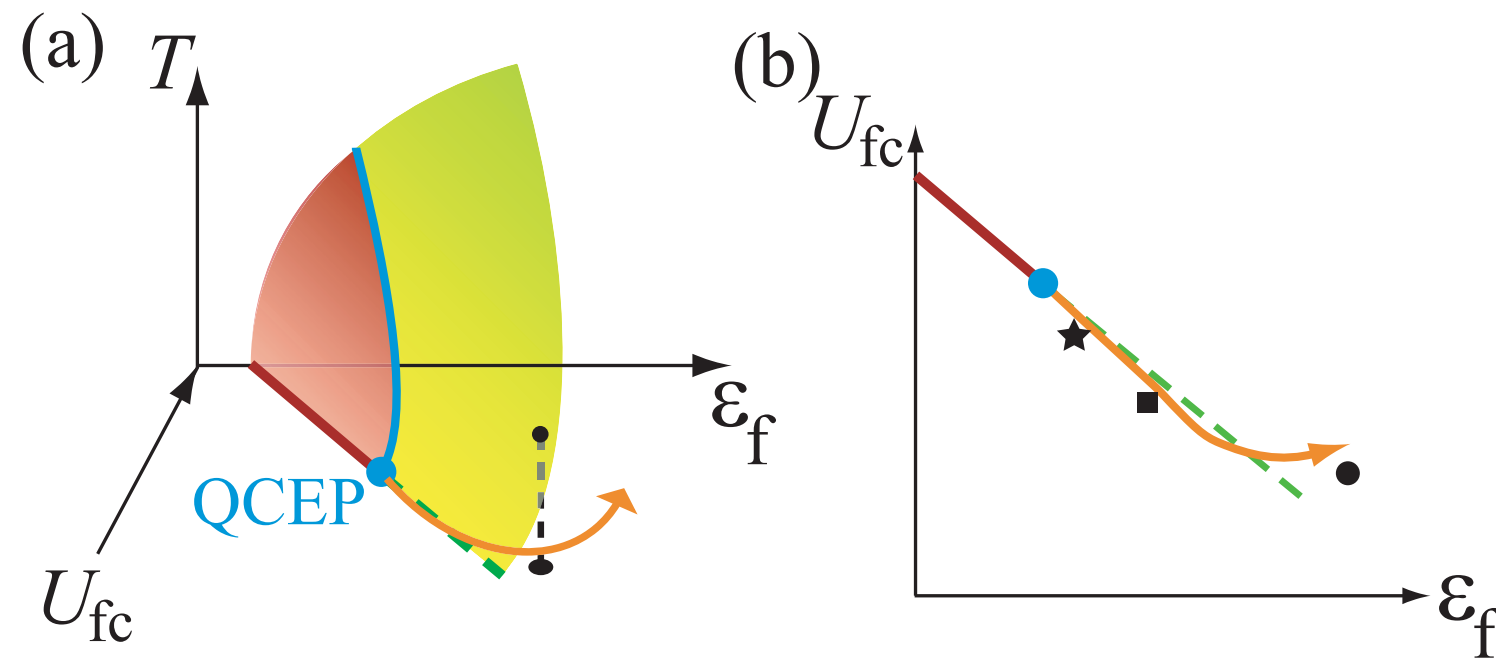

Figure 3. (color online) (a) Magnetic-field dependence of FOVT surface and valence-crossover surface in the $T-\varepsilon_{\mathrm{f}}-U_{\mathrm{fc}}$ phase diagram. The FOVT surface (dark surface) and the valencecrossover surface (light surface) are separated by the critical-end line (thick line) touched at $T=0 \mathrm{~K}$, which is the QCEP. The thick line with an arrow indicates the locus of the QCEP under magnetic field [22]. The black circle indicates a possible location of $\mathrm{YbAgCu}_{4}$ which touches the valence-crossover surface at about $T=40 \mathrm{~K}$ (black dashed line). (b) Ground-state phase diagram in $\varepsilon_{\mathrm{f}}-U_{\mathrm{fc}}$ plane. The thick line with an arrow represents the locus of the QCEP under a magnetic field. The black square and circle indicate possible locations of CeIrIn 5 and $\mathrm{YbAgCu}_{4}$, respectively (see text and also Ref. [38]). The black star indicates a possible location of $\mathrm{YbAuCu}_{4}$.

extension of the QCEP of the FOVT to the MV regime actually occurs, giving rise to the metamagnetisim [22]. This finding is quite important and hence we put special emphasis on this result: At the QCEP of the FOVT, not only the valence fluctuation, i.e., charge fluctuation, but also the magnetic susceptibility diverges.

Furthermore, this result indicates that even in the intermediate-valence materials, which does not show any valence transition at $H=0$, the QCEP of the FOVT is induced by applying the magnetic field $H$. An important point here is that as shown in Fig. 2(b), most of the Ce- and Yb-based compounds are located in the region for a moderate $U_{\mathrm{fc}}$, but not in the region for a large $U_{\mathrm{fc}}$ causing the FOVT. Hence, in the Ce- and Yb-based compounds, the valence-crossover temperature $T_{\mathrm{v}}^{*}(H)$ with strong valence fluctuations can emerge in the $T-H$ phase diagrams. Here, the magnitude of the characteristic field to make $T_{\mathrm{v}}^{*}(H)$ emerge depends on how close the location of the material is to the QCEP of the FOVT in the phase diagram. When the material is located close to the QCEP, metamagnetism as well as non-Fermi liquid behavior is expected to be observed by applying even a small magnetic field. In the following sections of $\S 3.2, \S 3.3$, and $\S 3.4$, we discuss the possible relevance of this mechanism to experimental observations.

\subsection{Metamagnesim and non-Fermi liquid behavior in CeIrIn ${ }_{5}$}

$\mathrm{CeIrIn}_{5}$ is a heavy-fermion metal, which shows a superconducting transition at $T=0.4 \mathrm{~K}$ at ambient pressure [23. When pressure is applied, the superconducting transition temperature increases in spite of the fact that the In-NQR relaxation rate $\left(T_{1} T\right)^{-1}$ drastically decreases, suggesting the possibility that a pairing mechanism different from the spin-fluctuation-mediated 


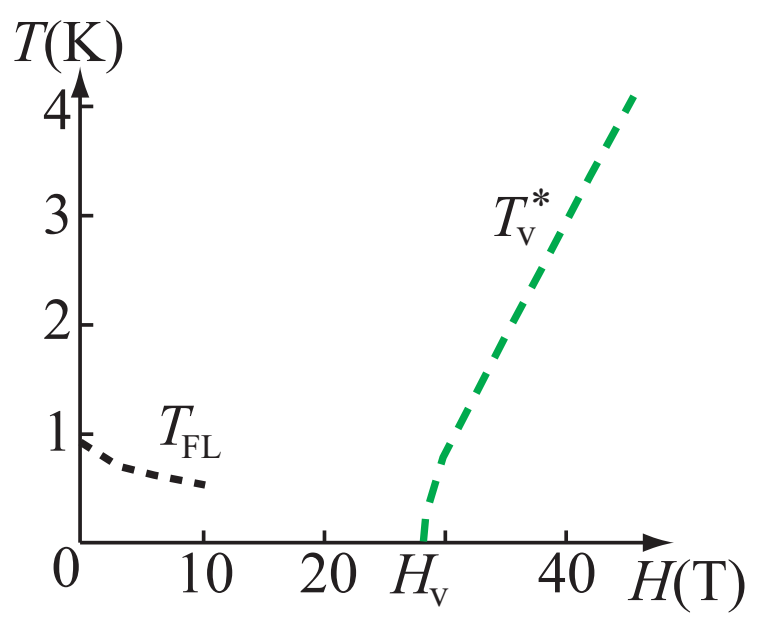

Figure 4. (color online) $T$ - $H$ phase diagram in CeIrIn 5 [29]. For $T<T_{\mathrm{FL}}$, Fermi-liquid behavior appears in specific heat data [28].

one is relevant to CeIrIn 5 24]. Interestingly, a metamagnetic anomaly was found to exist in the magnetization curve in CeIrIn ${ }_{5}$ 25. Several experimental groups have reported this anomaly occurring at the crossover (or first-order-transition) line in the $T-H$ phase diagram, as shown in Fig. 4 [26, 27, 28, 29]. As $H$ increases to approach $H_{\mathrm{v}}$, non Fermi-liquid behavior becomes prominent: (i) Convex behavior appears in the $T^{1.5}$ plot of the low- $T$ resistivity suggesting that the $T$-linear resisivity appears near $H=H_{\mathrm{v}}$ [28]. (ii) Residual resistivity has a peak at $H=H_{\mathrm{v}}[29$. (iii) The specific-heat constant shows a logarithmic divergence $C / T \sim-\log T$ near $H=H_{\mathrm{v}}[28$. The properties of (i) and (ii) are consistent with the theoretical results of critical valence fluctuations in Ref. [7] and Ref. [11, respectively, as explained in $\S 1$. The property of (iii) is also consistent with a theory recently developed for quantum valence criticality, which has shown that evaluation of the quasiparticle self-energy for one valence-fluctuation exchange process gives the low- $T$ specific heat as $C / T \sim-\log T$ in a certain- $T$ regime [30. In Fig. 4, the crossover line $T_{\mathrm{v}}^{*}(H)$ touches $T=0 \mathrm{~K}$ at $H=H_{\mathrm{v}} \sim 28 \mathrm{~T}[29$. This fact and the above observations of (i)-(iii) suggest that $\mathrm{CeIrIn}_{5}$ is located at the valence-crossover regime indicated by the black square in Fig. 3(b). At $H=0$, the black-square point is located at the Kondo regime. However, as $H$ increases, the slope of the valence crossover line (dashed line) becomes steeper, which crosses the black-square point at $H \sim 28 \mathrm{~T}$. This causes emergence of the valencecrossover line $T_{\mathrm{v}}^{*}(H)$ for $H \geq 28 \mathrm{~T}$, as shown in Fig. 4. Namely, CeIrIn 5 seems to be located at a distance of about $H=H_{\mathrm{v}} \sim 28 \mathrm{~T}$ from the valence-crossover line (dashed line in Fig. 3(b)), close to the QCEP at $H=0$. Hence, $H=H_{\mathrm{v}}$ at $T=0 \mathrm{~K}$ in Fig. 4 seems to be in close proximity to the QCEP, which affects the low- $T$ physics in CeIrIn 5 .

Recent measurement of the de Haas-van Alphen (dHvA) effect has confirmed that the dHvA frequencies are not substantially altered at $H=H_{\mathrm{v}}$ in Fig. 44 29]. This implies that the Fermisurface topology remains essentially the same between the $H<H_{\mathrm{v}}$ regime and the $H>H_{\mathrm{v}}$ regime. This is also consistent with the metamagnetism caused by the field-induced QCEP of the FOVT: As explained in detail in Fig. 2(a) in $\S 2.2$, the Fermi-surface volume basically does not change at the FOVT and valence crossover so long as a system remains in a paramagnetic state (Of course, in case of the FOVT (valence crossover), the lattice constant shows a discontinuous (continuous) change, which gives a change of the wave number itself). We also note that no evidence of the folded Brillouin Zone below and above $H_{\mathrm{v}}$ has been obtained by the dHvA measurement in CeIrIn 5 [29]. This result indicates that the field-induced AF-ordered phase is 
unlikely to be realized at $H_{\mathrm{v}}$, although the possibility of the magnetic-breakdown effect in the dHvA measurement should be carefully examined.

We also note that CeIrIn 5 and $\mathrm{CeCoIn}_{5}$ have almost the same Fermi surfaces according to the band-structure calculations 31 and the dHvA measurements 32. Both materials have a quite similar level scheme of the crystalline electric field (CEF) [33. However, in $\mathrm{CeCoIn}_{5}$, the crossover line (or first-order transition line) accompanied by the non-Fermi liquid behavior as shown in Fig. 4 does not appear in the $T-H$ phase diagram. We note here that by applying pressure to $\mathrm{CeCoIn}_{5}$, residual resistivity and the $T^{2}$ coefficient $A$ of the low- $T$ resistivity drop drastically [34]. These observations suggest that the QCEP of the FOVT, or the sharp valence crossover point exists on a slightly-negative pressure side in the $T$ - $P$ phase diagram of $\mathrm{CeCoIn}_{5}$. Actually, the experimental fact of the emergence of the $T$-linear resistivity and logarithmic divergence of the specific heat $C / T \sim-\log T$ at low temperatures and ambient pressure in $\mathrm{CeCoIn}_{5}$ does not contradict this point of view [30].

These results indicate that a viewpoint of the closeness to the QCEP of the FOVT is important for understanding the Ce115 systems in addition to the conventional view based on the competition between the Kondo effect and the RKKY interaction. Indeed, this viewpoint offers us a key to resolving the outstanding puzzle in $\mathrm{CeRhIn}_{5}$ as well, which will be discussed in $\S 4.1$. Since the superconducting correlation has been shown to develop near the QCEP of the FOVT theoretically (see Figs. 2(a) and 3(b)) [10, 12, the proximity to the QCEP seems to control the occurrence of the unconventional superconductivity in $\mathrm{CeIrIn}_{5}$.

To examine our theoretical proposal, it is desirable to measure the valence change of Ce at the crossover line $T_{\mathrm{v}}^{*}$ (or first-order transition line) in Fig. 4. The X-ray adsorption spectra for direct observation of the Ce valence, the NQR measurement for the electric-field gradient, the ultrasonic measurement, and the X-ray diffraction and/or thermal expansion measurement for the lattice-constant change are highly desirable.

\subsection{Enhanced magnetic susceptibility and metamagnetism in $\mathrm{YbAgCu}_{4}$}

As noted in $\S 1, \mathrm{YbInCu}_{4}$ shows the FOVT at $T=T_{\mathrm{v}}=42 \mathrm{~K}[3$, 4]. When In is replaced by other elements, the FOVT has not been observed. However, anomalous behavior which seems to be related to valence fluctuations has been observed in $\mathrm{YbAgCu}_{4}$ 35. In $\mathrm{YbAgCu}_{4}$, uniform magnetic susceptibility has a broad peak at $T=40 \mathrm{~K}$. Below $T=40 \mathrm{~K}$, the volume expansion occurs [36] simultaneously with a sharp valence crossover from $\mathrm{Yb}^{+2.89}$ for $T>40 \mathrm{~K}$ to $\mathrm{Yb}^{+2.87}$ for $T<40 \mathrm{~K}$ [35], indicating that negative volume expansion occurs as $T$ increases to approach $T=40 \mathrm{~K}$. Namely, the uniform magnetic susceptibility is enhanced at the valence-crossover temperature $T=T_{\mathrm{v}}^{*}=40 \mathrm{~K}$ with strong valence fluctuations. This is consistent with our theoretical result discussed in $\S 3.1$ [18. At the QCEP and the critical-end line in Fig. 3(a), the valence fluctuations diverge. At the same time, the magnetic susceptibility diverges [22]. $\mathrm{YbAgCu}_{4}$ seems to be located in the valence-crossover regime indicated by the black circle in Fig. 3(a), whose distance from the valence-crossover surface at $H=0$ is about $T=40 \mathrm{~K}$ as shown by the black dashed line in Fig. 33(a) [18, 17]. Hence, the broad peak of the magnetic susceptibility is considered to be caused by the valence fluctuations developed at the valence-crossover surface. $\mathrm{YbAgCu}_{4}$ shows a metamagnetism in the low temperature limit around $H=40 \mathrm{~T}$ [35. This can also be naturally understood from the field-induced valence-crossover surface shown in Fig. 3(a). Namely, the location of $\mathrm{YbAgCu}_{4}$ indicated by the black circle in Fig. 3(b) is at a distance of about $H \sim 40 \mathrm{~T}$ from the valence-crossover line close to the QCEP at $H=0$. For more details, the readers can refer to Refs. [17] and [18.

\subsection{Enhanced magnetic susceptibility and metamagnetism in $Y_{b}{ }_{2} Z_{2} n_{20}$}

Recently, behaviors similar to those of $\mathrm{YbAgCu}_{4}$ have been observed in $\mathrm{YbIr}_{2} \mathrm{Zn}_{20}$ [37. The uniform magnetic susceptibility has a peak at $T=T_{\mathrm{v}}^{*}=7.4 \mathrm{~K}$ accompanied by the volume 
expansion for $T<T_{\mathrm{v}}^{*}$. Since the unit-cell volume with the $\mathrm{Yb}^{+2.0}\left(4 \mathrm{f}^{0}\right.$ hole configuration) state is larger than that with the $\mathrm{Yb}^{+3.0}\left(4 \mathrm{f}^{1}\right.$ hole configuration) state, this observation suggests that the enhancement of the magnetic susceptibility is caused by the enhanced Yb-valence fluctuations associated with the valence change of $\mathrm{Yb}$. This viewpoint is consistent with the fact that the thermal expansion coefficient is negative for $T<T_{\mathrm{v}}^{*}$ [37.

Recently, it has been found that $\mathrm{YbIr}_{2} \mathrm{Zn}_{20}$ shows a metamagnetism at $H \sim H_{\mathrm{m}}=10 \mathrm{~T}$ [37. Near the metamagnetic field $H_{\mathrm{m}}$, enhancement of the cyclotron mass of electrons has been observed by the dHvA measurement. The specific-heat constant $C / T$ and the $T^{2}$ coefficient of the resistivity at low temperatures also indicate the mass enhancement of electrons at $H=H_{\mathrm{m}}$. Furthermore, residual resistivity has a peak at $H=H_{\mathrm{m}}$. The temperature region where the low- $T$ resistivity shows the $T^{2}$ dependence becomes narrowest in the vicinity of $H=H_{\mathrm{m}}$, which suggests a tendency of the $T$-linear resistivity near $H=H_{\mathrm{m}}$. All these observations are naturally explained by the field-induced valence crossover discussed in $\S 3.1$. Although the mass enhancement and the residual-resistivity peak suggest a clear signature of the Yb-valence fluctuations developed when $H$ approaches $H_{\mathrm{m}}$, the experimental fact that the resistivity has the $T^{2}$ dependence at the lowest temperatures even at $H=H_{\mathrm{m}}$ implies that $\mathrm{YbIr}_{2} \mathrm{Zn}_{20}$ at ambient pressure is located at the valence-crossover regime. Namely, $\mathrm{YbIr}_{2} \mathrm{Zn}_{20}$ seems to be located at a similar position to that of $\mathrm{YbAgCu}_{4}$, indicated by the black circle in Fig. 3(b). The distance from the valence-crossover line close to the QCEP at $H=0$ seems to be about $H \sim 10 \mathrm{~T}$ in $\mathrm{YbIr}_{2} \mathrm{Zn}_{20}$, since metamagnetic field is about $H_{\mathrm{m}} \sim 10 \mathrm{~T}$ [38. Indeed, a steep volume shrinkage has been observed at $H \sim H_{\mathrm{m}}$ [37, which indicates that the Yb valence sharply increases at $H=H_{\mathrm{m}}$. The dHvA measurement has concluded that the Fermi surfaces do not change at $H=H_{\mathrm{m}}$ from the fact that the dHvA frequencies do not change across $H_{\mathrm{m}}$ [37. This is also consistent with the mechanism of the field-induced valence crossover, since the Fermi-surface volume is essentially unchanged at the valence crossover so long as the system remains in the paramagnetic state as discussed in $\S 2.2$ and also in $\S 3.2$.

When pressure is applied to $\mathrm{YbIr}_{2} \mathrm{Zn}_{20}$, the metamagnetic field $H_{\mathrm{m}}$ can be tuned to approach $H=0 \mathrm{~T}\left[39\right.$. This seems to be consistent with the location of $\mathrm{YbIr}_{2} \mathrm{Zn}_{20}$ mentioned above, since applying pressure makes $U_{\mathrm{fc}}$ large because of the reduction of the distance between wavefunctions of $4 \mathrm{f}$ and conduction electrons and also makes $\varepsilon_{\mathrm{f}}<0$ small (i.e., $\left|\varepsilon_{\mathrm{f}}\right|$ large) in the hole picture. Here, we should note that the panel of the cutout shown in Fig. 2(b) is drawn for the Ce compounds. In the case of $\mathrm{Yb}$ compounds, the panel tilts with a certain angle with the opposite sign to the $\varepsilon_{\mathrm{f}}$ axis. The observation of the quantum criticality at the QCEP of the FOVT by tuning the control parameters of the pressure and/or magnetic field is an interesting future issue [0]. Systematic measurements in $\mathrm{YbX}_{2} \mathrm{Zn}_{20}(\mathrm{X}=\mathrm{Ir}, \mathrm{Co}$, and $\mathrm{Rh})$ are highly desirable for a unified understanding of the effect of the critical valence fluctuations.

\section{Interplay of magnetic order and valence fluctuations}

In the preceding sections, we have discussed the nature of the QCEP of the FOVT in a paramagnetic phase and how it is controlled by applying the pressure and/or magnetic field. In Ce- and Yb-based heavy fermion systems, however, the magnetic order can also occur. The interplay of the magnetic order and Ce- or Yb-valence fluctuations offers a key concept for understanding these systems. Below we focus on $\mathrm{CeRhIn}_{5}$ as a prototypical material to demonstrate how such an interplay plays a crucial role in understanding the puzzling behaviors.

\section{1. $\mathrm{CeRhIn}_{5}$}

$\mathrm{CeRhIn}_{5}$ is a heavy-fermion metal [41] which undergoes an $\mathrm{AF}$ transition at $T_{\mathrm{N}}=3.8 \mathrm{~K}$ with the ordered vector $\mathbf{Q}=(1 / 2,1 / 2,0.297)$ at ambient pressure [42]. When pressure is applied, the AF-ordered phase changes to the paramagnetic and superconducting phase at $P=P_{\mathrm{c}} \sim 2 \mathrm{GPa}$, as shown schematically in Fig. 5 [43, 44, 45, 46, 47, 48, 49]. This material has attracted much 


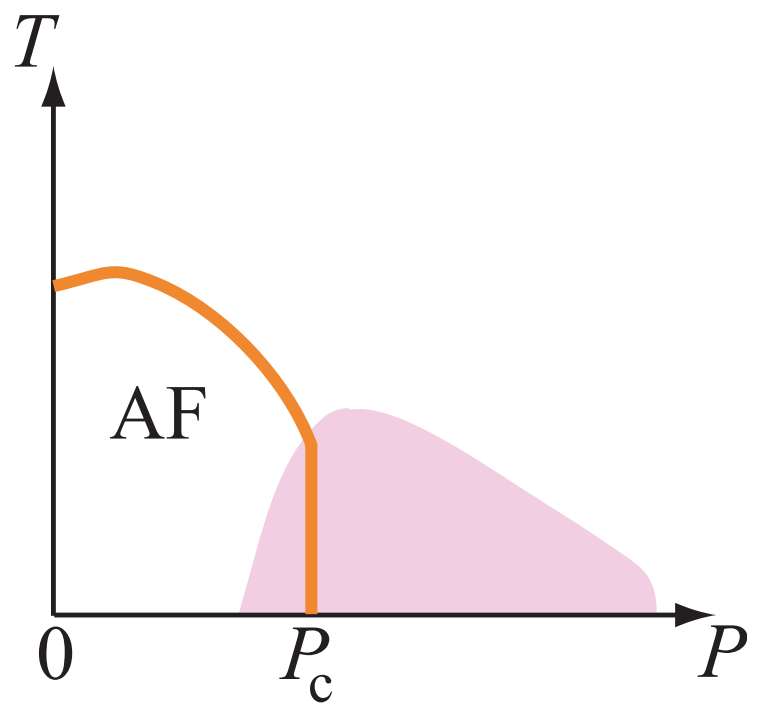

Figure 5. (color online) Schematic $T-P$ phase diagram of $\mathrm{CeRhIn}_{5}$ [43, 44]. Shaded region represents superconducting phase.

attention since accumulated experiments offer outstanding puzzle, whose significant features are summarized as follows: (i) The Sommerfeld constant $\gamma_{\mathrm{e}} \sim 50 \mathrm{~mJ} /\left(\mathrm{molK}^{2}\right)$ in the AF state at $P=0$ is about 10-times larger than $\gamma_{\mathrm{e}}=5.7 \mathrm{~mJ} /\left(\mathrm{molK}^{2}\right)$ in $\operatorname{LaRhIn}_{5}$ [41, 46]. (ii) The Fermi surfaces similar to those in $\mathrm{LaRhIn}_{5}$ in the AF phase for $P<P_{\mathrm{c}} \sim 2.35 \mathrm{GPa}$ change to the Fermi surfaces similar to those in $\mathrm{CeCoIn}_{5}$ in the paramagnetic phase for $P>P_{\mathrm{c}}$ by the dHvA measurement performed under the magnetic field $H \sim 15 \mathrm{~T}$ [46]. (iii) The cyclotron mass of electrons shows an enhancement toward $P=P_{\mathrm{c}}$ : The cyclotron mass of the $\beta_{2}$ branch, whose Fermi surface has a cylindrical shape, changes from $6 m_{0}$ at $P=0$ to $60 m_{0}$ at $P \lesssim P_{\mathrm{c}}$ and the signal is not detected for $P>P_{\mathrm{c}}$, probably because of a too heavy mass, $\sim 100 m_{0}$ [46]. (iv) The $T$-linear resistivity emerges prominently near $P=P_{\mathrm{c}}$ [43, 44, 47]. (v) Residual resistivity has a peak at $P \sim P_{\mathrm{c}}$ 43, 44, 47]. (vi) The superconducting phase exists in a wide pressure region around $P=P_{\mathrm{c}}$ [43, 44, 45].

Because of the dHvA measurement noted above (ii), one might succumb to the temptation to believe a scenario in which the localized to itinerant transition of $\mathrm{f}$ electrons happens at $P=P_{\mathrm{c}}$ [50, 51]. However, this scenario encounters a serious difficulty in explaining the experimental fact (i) above: The AF state with the 10-times mass enhancement at $P=0$ strongly suggests that the heavy quasiparticles contribute to the formation of the AF state, indicating the existence of the c-f hybridization even in the AF phase for $P<P_{\mathrm{c}}$. Hence, the theoretical explanation for resolving a series of puzzles outlined above (i)-(vi) in a natural and unified way has been desired.

First of all, let us point out that the transport anomalies above (iv) and (v) and the robust superconducting phase (vi) are quite similar to the observations in the $\mathrm{CeCu}_{2}(\mathrm{Si} / \mathrm{Ge})_{2}$ systems [6, 7, 8] introduced in $\S 1$. Emergence of the $T$-linear resistivity prominent at $P=P_{\mathrm{v}}$ in Fig. 1(b), at which the residual resistivity has a peak, covered with the superconducting phase in the wide pressure region is the common feature in $\mathrm{CeCu}_{2} \mathrm{Ge}_{2}$ [6], $\mathrm{CeCu}_{2} \mathrm{Si}_{2}$ [7], and $\mathrm{CeCu}_{2}\left(\mathrm{Ge}_{x} \mathrm{Si}_{1-x}\right)_{2}$ [8]. Since the $T$-linear resistivity and residual resistivity peak appear at the AF-paramagnetic boundary in CeRhIn $5, P_{\mathrm{c}} \approx P_{\mathrm{v}}$ seems to be realized in Fig. 5. As will be shown below, this is actually the case realized in the model Eq. (11) for realistic parameters for 
(a)

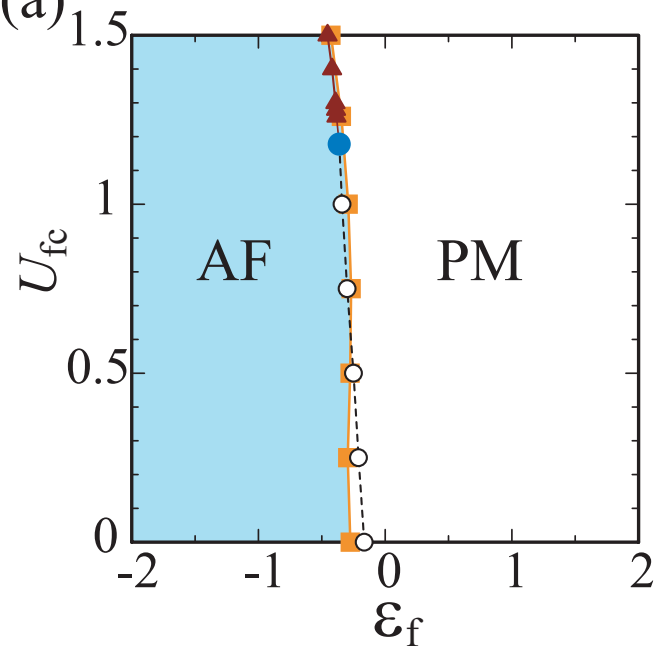

(b)

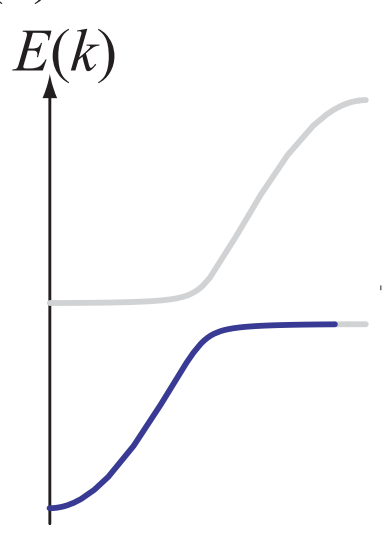

(c)

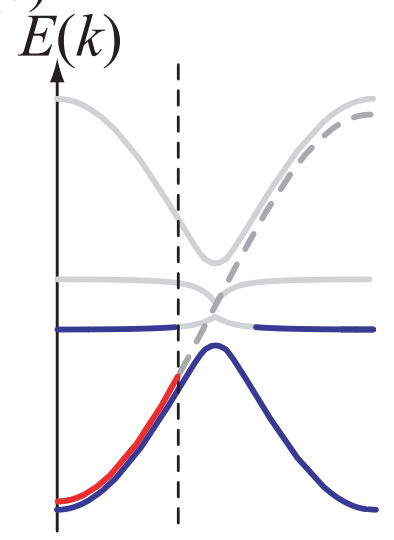

Figure 6. (color online) (a) Ground state phase diagram in $\varepsilon_{\mathrm{f}}-U_{\mathrm{fc}}$ plane of the model Eq. (11) for $V=0.2$ and $U=\infty$ at $n=0.9$ (see text) 21. The FOVT (solid line with triangles) terminates at QCEP (filled circle). Valence crossover with enhanced valence fluctuations occurs at the dashed line with open circles. The solid line with filled squares represents the antiferromagnetic (AF)-paramagnetic (PM) boundary. Occupied (solid lines) and empty (gray lines) bands in the periodic Anderson model at $n=\left(n_{\mathrm{f}}+n_{\mathrm{c}}\right) / 2=0.9$ are shown in the paramagnetic phase (b) and in the AF-ordered phase (c). In (c), the gray dashed line indicates the energy band of the conduction band, $\varepsilon_{\mathbf{k}}$ at $n_{\mathrm{c}}=0.8$. The black dashed line is a guide for the eyes indicating that the Fermi surface in AF-ordered phase with finite c-f hybridization coincides with the small Fermi surface where f electrons are completely localized.

$\mathrm{CeRhIn}_{5}$, which naturally resolves the above puzzles (i)-(vi) [21].

Figure 6(a) shows the ground-state phase diagram of the model Eq. (1) without the magnetic field on the square lattice obtained by the slave-boson mean-field theory [52], which enables us to treat the $\mathrm{AF}$ order and valence transition or fluctuations on equal footing. Here, we set small hybridization $V=0.2$ and the conduction band as $\varepsilon_{\mathbf{k}}=-2 t\left(\cos k_{x}+\cos k_{y}\right)$ at the total filling $n=0.9$ to mimic the $\beta_{2}$ branch of $\mathrm{CeRhIn}_{5}$ [46]. The FOVT line (solid line with triangles) terminates at the QCEP (filled circle). Valence crossover with enhanced valence fluctuations occurs on the dashed line with open circles. These are the results within the paramagnetic states. When the AF order with the ordered vector $\mathbf{Q}=(\pi, \pi)$ is taken into account, we find that the AF-paramagnetic boundary represented by the solid line with filled squares emerges at almost the same place as the FOVT line and the valence-crossover line. Since $\varepsilon_{\mathrm{f}}$ can be regarded as the pressure as explained in $\S 2.3$, this result implies that $P_{\mathrm{c}} \approx P_{\mathrm{v}}$ is realized.

The precise explanation of this mechanism is illustrated in Figs. Z(a) and 7(b). As shown in Fig. 7(a), when the Néel temperature close to $0 \mathrm{~K}$, i.e., $T_{\mathrm{N}}(P) \sim 0 \mathrm{~K}$, meets the valencecrossover temperature $T_{\mathrm{v}}^{*}(P)$, enhanced valence fluctuations suppress the AF order. Then, the AF order is suddenly cut around $P=P_{\mathrm{v}}$, as shown in Fig. 7(b). Actually, the slave-boson mean-field calculation shows that the AF order exhibits the first-order transition at the solid line with filled squares in Fig. 6(a) [21]. Namely, the coincidence of the AF transition and the valence crossover or transition is caused by the enhanced valence fluctuations or the FOVT. Hence, the $P_{\mathrm{c}} \approx P_{\mathrm{v}}$ illustrated in Fig. 1(c), or more precisely in Fig. 7(b), is shown to be realized for a small hybridization case in the model Eq. (1), which is a realistic parameter for 

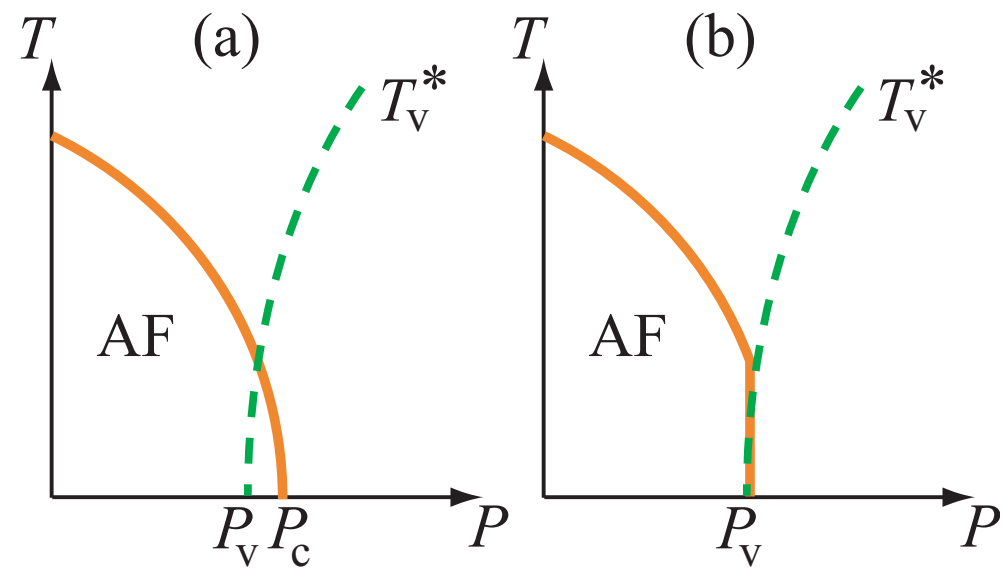

Figure 7. (color online) (a) Schematic $T$ - $P$ phase diagram for $P_{\mathrm{v}}<P_{\mathrm{c}}$. (b) Enhanced valence fluctuations at $P_{\mathrm{v}}$ suppress the $\mathrm{AF}$ order, giving rise to the $\mathrm{AF}$ transition of the first order at $P=P_{\mathrm{v}}$ shown by vertical thick line [21].

$\mathrm{CeRhIn}_{5}$ [46]. We should remark that for larger hybridization $V$, the AF-paramagnetic boundary appears at smaller $\varepsilon_{\mathrm{f}}$ than the FOVT and valence-crossover line in Fig. 6(a), which corresponds to Fig. 1(b). Hence, depending on the strength of the hybridization $V$, the relative position of $P_{\mathrm{c}}$ and $P_{\mathrm{v}}$ changes as shown in Figs. 1(b) and 1(c), and Fig. 7(a).

We have also shown that the Fermi surface calculated for $V=0.2$ in the AF phase at $n=\left(n_{\mathrm{f}}+n_{\mathrm{c}}\right) / 2=0.9$ is nearly the same as the small Fermi surface without c-f hybridization where electrons for $n_{\mathrm{f}}=1$ are located at the localized $\mathrm{f}$ level and the conduction band is filled up with all extra electrons for $n_{\mathrm{c}}=0.8$ [21]. This naturally explains the dHvA measurement (ii) above. In the paramagnetic phase in Fig. 6(a), the large Fermi surface, which counts the f-electron number in the total Fermi volume, i.e., $n_{\mathrm{f}}+n_{\mathrm{c}}$, is realized, as shown in Fig. 6(b). In the AF phase, the lower and upper hybridized bands are folded, as shown in Fig. 6(c). Since the lower folded hybridized band is completely filled, the Fermi surface becomes the same as that of the conduction electrons at the filling $n_{\mathrm{c}}$. Hence, we stress that the small Fermi surface appears in the AF phase with finite $c$-f hybridization, $\left\langle f_{\mathbf{k} \sigma}^{\dagger} c_{\mathbf{k} \sigma}\right\rangle \neq 0$.

The mass enhancement observed by the dHvA measurement (iii) above is also quantitatively reproduced by the same model Eq. (1) under the magnetic field $H=15 \mathrm{~T}$ [21]. Namely, the mass enhancement of the two-dimensional-like Fermi surface of the $\beta_{2}$ branch from $6 m_{0}$ at $P=0$ to $60 m_{0}$ at $P \lesssim P_{\mathrm{c}}[46$ ] is well reproduced by the present mechanism, which shows that the total density of states at the Fermi level increases near the AF-paramagnetic boundary in Fig. [6(a). This is because when the pressure is applied to the AF state, i.e., when $\varepsilon_{\mathrm{f}}$ increases, the gap between the original lower hybridized band and the folded band increases as in Fig. 6(c). Then, the f-electron-dominant flat part of the folded lower hybridized band approaches the Fermi level, giving rise to the increase in the density of states. In the paramagnetic phase, as $\varepsilon_{\mathrm{f}}$ approaches the AF-paramagnetic boundary in Fig. 6(a), i.e., as $\varepsilon_{\mathrm{f}}$ decreases, $n_{\mathrm{f}}$ increases to approach 1 , which is in the so called Kondo regime. Hence, the density of states at the Fermi level increases. Thus, the reason why the mass enhancement occurs toward the AF-paramagnetic boundary $P=P_{\mathrm{c}}$ in $\mathrm{CeRhIn}_{5}$ is naturally explained.

This result is quite consistent with a recent experiment on the effective mass of electrons [43]: Knebel et al applied a magnetic field to $\mathrm{CeRhIn}_{5}$, whose magnitude is the same as $H=15 \mathrm{~T}$ used in the dHvA measurement [46]. After the disappearance of the superconductivity, the $T^{2}$ 
dependence of the resistivity appears at lowest temperatures. Knebel et al found that the $\sqrt{A}$ with $A$ being the coefficient of the $T^{2}$ term scales nicely with the cyclotron mass of the $\beta_{2}$ branch under pressure for $0 \leq P \leq P_{\mathrm{c}}$, i.e., $\sqrt{A} / m^{*}=$ const. scaling holds [43]. This scaling indicates that the mass enhancement toward $P=P_{\mathrm{c}}$ is essentially caused by the effect of the energy band dispersion of quasiparticles [53]. We also note that about 10-times mass enhancement at $P=0$ is also reproduced by the same model Eq. (1) 21], which is quite consistent with the experimental fact (i) above. Formation of the heavy quasiparticles via the $c-f$ hybridization naturally explains the mass enhancement even inside of the AF phase.

As shown in Fig. 6(a), $P_{\mathrm{c}} \approx P_{\mathrm{v}}$ is a consequence of the suppression of the AF order by enhanced valence fluctuations for moderate $U_{\mathrm{fc}}$, e.g., $U_{\mathrm{fc}} \approx 0.5$, which is a realistic parameter for $\mathrm{CeRhIn}_{5}$, giving rise to the $T$-linear resistivity and the residual resistivity peak at the AFparamagnetic boundary. Hence, the experimental facts (iv) and (v) are naturally explained. Since the superconducting phase was shown to be realized around $P_{\mathrm{v}}[10$, 12, as shown in Fig. 1(b), the above (vi) is also consistently explained (see Fig. 5 and Fig. 7(b)). Hence, all of the above experimental observations (i)-(vi) are naturally explained in a unified way.

We note that local correlation effects of $4 \mathrm{f}$ electrons give rise to the large ordered moment of the AF order: Only about $10 \%$ reduction of the full ordered moment for the Kramers doublet of the lowest $\mathrm{CEF}$ level is realized even near the AF-paramagnetic boundary in Fig. 6(a) by the slave-boson mean-field theory [21], which is consistent with the observation in $\mathrm{CeRhIn}_{5}$ [48]. Our result shows that strong onsite Coulomb repulsion of $4 \mathrm{f}$ electrons yields the local-moment-like behavior as if $\mathrm{f}$ electrons are localized, in spite of the fact that $\mathrm{f}$ electrons are always itinerant, i.e., $\left\langle f_{\mathbf{k} \sigma}^{\dagger} c_{\mathbf{k} \sigma}\right\rangle \neq 0$ always holds everywhere of the phase diagram shown in Fig. 6)(a), which is nothing but a manifestation of the localized-itinerant duality [54].

As reported in Ref. [55], the experimental comparison between $\mathrm{CeRhIn}_{5}$ and $\mathrm{CeCo}\left(\operatorname{In}_{1-x} \mathrm{Cd}_{x}\right)_{5}$ shows that $\mathrm{CeRhIn}_{5}$ at $P \gtrsim P_{\mathrm{c}}$ corresponds to CeCoIn 5 at $P=0$ [16]. This is quite consistent with our argument in $\S 3.2$ : Because $P_{\mathrm{c}} \approx P_{\mathrm{v}}$ is realized in $\mathrm{CeRhIn}_{5}$, if the above correspondence holds, that implies that on the slightly-negative pressure side in the $T$ - $P$ phase diagram of $\mathrm{CeCoIn}_{5}$, the QCEP or sharp Ce-valence crossover point, i.e., $P=P_{\mathrm{v}}$, exists, which is the same conclusion stated in $§ 3.2$.

The interplay of the magnetic order and enhanced valence fluctuations plays an important role not only in $\mathrm{CeRhIn}_{5}$ but also in other materials. One such example is $\mathrm{YbAuCu}_{4}$, which will be discussed in the next section.

\section{2. $\mathrm{YbAuCu}_{4}$}

$\mathrm{YbAuCu}_{4}$ is a sister compound of $\mathrm{YbInCu}_{4}$, which exhibits the FOVT as noted in $\S 1$. We note that the phase diagram of $\mathrm{YbAuCu}_{4}$ shown in Fig. 8 is essentially the same as Fig. 1(c) or Fig. 7(b), if $P$ is replaced by $H\left[56\right.$. The ${ }^{63} \mathrm{Cu}-\mathrm{NQR}$ measurement has detected that there exists a characteristic temperature $T_{\mathrm{v}}^{*}(H)$ in the $T$ - $H$ phase diagram, at which the $\mathrm{Cu}-\mathrm{NQR}$ frequency changes sharply, strongly suggesting that the $\mathrm{Yb}$-valence crossover occurs [57]. The Yb-valence crossover temperature $T_{\mathrm{v}}^{*}(H)$ becomes $T_{\mathrm{v}}^{*}=0 \mathrm{~K}$ at the field $H_{\mathrm{v}}$, which seems to coincide with the field $H_{\mathrm{c}}$ at which the Néel temperature goes to $T_{\mathrm{N}}=0 \mathrm{~K}$ [56, 57] (see Fig. 8). Namely, $H_{\mathrm{c}} \approx H_{\mathrm{v}}$ occurs in the $T-H$ phase diagram. As discussed in $\S 3.1$, the valence crossover temperature $T_{\mathrm{v}}^{*}(H)$ is induced by applying the magnetic field. If $\mathrm{YbAuCu}_{4}$ is located at the moderate $U_{\mathrm{fc}}$ regime indicated by the black star in Fig. 3(b), the appearance of $T_{\mathrm{v}}^{*}(H)$ in Fig. 8 is naturally explained [17]. Namely, $\mathrm{YbAuCu}_{4}$ seems to be in the Kondo regime at $H=0$ and by applying $H=H_{\mathrm{v}} \sim 1.3 \mathrm{~T}$, the valence crossover $T_{\mathrm{v}}^{*}(H)$ is induced, as shown in Fig. 8. This indicates that $\mathrm{YbAuCu}_{4}$ is close to the QCEP at $H=0$ with a distance of about $H \sim 1.3 \mathrm{~T}$ as indicated by the black star in Fig. 3(b). As explained in $\S 4.1$, when $T_{\mathrm{N}}(H)$ close to $0 \mathrm{~K}$ meets $T_{\mathrm{v}}^{*}(H)$, a suppression of the magnetic order by enhanced valence fluctuations occurs, which gives rise to the coincidence $H_{\mathrm{c}} \approx H_{\mathrm{v}}$. In $\mathrm{YbAuCu}_{4}$, the cusp-like anomaly of the residual resistivity 


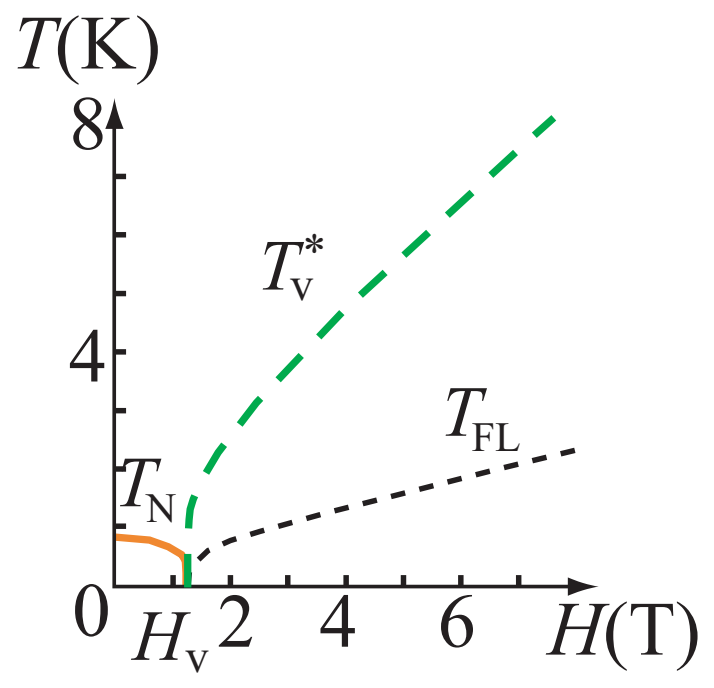

Figure 8. (color online) $T-H$ phase diagram of $\mathrm{YbAuCu}_{4}$ [57. The Néel temperature $T_{\mathrm{N}}$ is suppressed and the $\mathrm{Yb}$ valence crossover temperature $T_{\mathrm{v}}^{*}$ (thick dashed line) is induced for $H>H_{\mathrm{v}} \sim 1.3 \mathrm{~T}$. For $T<T_{\mathrm{FL}}$ (black dashed line), the resistivity shows the Fermi liquid behavior $\rho \sim T^{2}$.

was observed at $H=H_{\mathrm{c}} \approx H_{\mathrm{v}}$ [57]. The mass enhancement of electrons estimated from the $A$ coefficient in the resistivity was also observed near $H=H_{\mathrm{c}} \approx H_{\mathrm{v}}$ [57]. These observations are naturally explained by the enhanced Yb-valence fluctuations [17].

\section{Summary and outlook}

In this article, the roles of critical valence fluctuations in Ce- and Yb-based heavy fermion metals are discussed. Recent development of theory and experiment has revealed that critical Ce- and $\mathrm{Yb}$-valence fluctuations play a key role in several anomalies in this family of materials. Magnetic field is shown to be a useful control parameter to induce the quantum critical end point (QCEP) of the first-order valence transition (FOVT). As a prototypical material, metamagnetism and non-Fermi liquid behavior in CeIrIn $5, \mathrm{YbAgCu}_{4}$, and $\mathrm{YbIr}_{2} \mathrm{Zn}_{20}$ are shown to be naturally explained by the mechanism of the field-induced valence crossover. The interplay of the magnetic order and Ce- or Yb-valence fluctuations is a key mechanism for understanding anomalous behavior in Ce- and Yb-based heavy fermion systems. It is shown that the interplay resolves the outstanding puzzle in $\mathrm{CeRhIn}_{5}$. The origin of the transport anomalies and drastic change of the Fermi surfaces accompanied by the mass enhancement of electrons is naturally explained by the interplay in a unified way. A special emphasis is put on the fact that a small Fermi surface generally appears by the folding of the hybridized band in the AF phase in the periodic Anderson model. The unified explanation for $\mathrm{CeInIn}_{5}$ and $\mathrm{CeRhIn}_{5}$ strongly suggests that the viewpoint of the closeness to the QCEP of the FOVT is indispensable in understanding the Ce115 systems. As another prototypical material where the interplay occurs, the $T-H$ phase diagram of $\mathrm{YbAuCu}_{4}$ is discussed. The proximity to the QCEP of the FOVT is a key concept for understanding Ce- and Yb-based heavy fermion systems as an underling mechanism.

For outlook, recently synthesized materials, $\mathrm{YbIr}_{2} \mathrm{Zn}_{20}, \mathrm{YbCo}_{2} \mathrm{Zn}_{20}$, and $\mathrm{YbRh}_{2} \mathrm{Zn}_{20}$ are promising materials, which are expected to show the properties caused by the Yb-valence fluctuations by tuning the control parameters of the magnetic field and pressure. Recently, in $\beta$-YbAlB ${ }_{4}$, where the superconductivity was first discovered among Yb-based compounds [61, 
evidence of strong Yb-valence fluctuations has been reported [62]. Further study to clarify the role of the valence fluctuations in the unconventional criticality as well as the origin of the superconductivity is an interesting future problem. We point out that the $T-H$ phase diagram in $\mathrm{YbRh}_{2} \mathrm{Si}_{2}$ [58], where unconventional criticality has attracted much attention in correlated electron systems [59, is closely similar to that in $\mathrm{YbAuCu}_{4}$ (see Fig. 8). Hence, there exists a possibility that the critical $\mathrm{Yb}$-valence fluctuation plays a key role in the origin of the unconventional criticality in $\mathrm{YbRh}_{2} \mathrm{Si}_{2}$. Thus, it is quite important to examine the possibility of whether the $\mathrm{Yb}$-valence change takes place at the crossover temperature $T^{*}(H)$ whose origin has not yet been clarified experimentally in the $T-H$ phase diagram. The experimental fact that $\mathrm{YbRh}_{2} \mathrm{Si}_{2}$ shows a huge mass enhancement even at $H=0$ such as $\gamma_{\mathrm{e}} \sim 1.7 \mathrm{~J} /\left(\mathrm{molK}^{2}\right)$ deep inside of the AF phase [60] indicates that heavy quasi particles form the AF-ordered state as in $\mathrm{CeRhIn}_{5}$. The Co-NQR measurement in $\mathrm{Yb}\left(\mathrm{Rh}_{1-x} \mathrm{Co}_{x}\right)_{2} \mathrm{Si}_{2}$ and/or the direct observation of the $\mathrm{Yb}$ valence by X-ray adsorption measurement at the crossover temperature $T^{*}(H)$ are highly desirable. As noted in $\S 2.3$, the magnitude of the valence change is expected to be of the order of 0.01 in most of the $\mathrm{Ce}$ and $\mathrm{Yb}$ compounds. Hence, high accuracy measurement which can detect the tiny change of the valence is a challenging future problem, which will open new avenues of study in this field.

\section{Acknowledgements}

This work is supported by the Global COE program (G10) and a Grant-in-Aid for Scientific Research (No. 19340099) from the Japan Society for the Promotion of Science (JSPS), and a Grant-in Aid for Scientific Research on Innovative Areas "Heavy Electrons" @(No.20102008) from the Ministry of Education, Culture, Sports, Science and Technology of Japan. S. W. is supported by a Grant-in-Aid for Young Scientists (B) (No. 21740240) from JSPS.

\section{References}

[1] Gschneidner K A and Eyring L, Handbook on the Physics and Chemistry of Rare Earths (North-Holland, Amsterdam, 1978)

[2] Wohlleben D and Rohler J, 1984 J. Appl. Phys. 5515

[3] Felner I and Nowik I 1986 Phys. Rev. B 33617

[4] J. L. Sarrao, C. L. Benton, Z. Fisk, J. M. Lawrence, D. Mandrus, and J. D. Thompson 1996 Physica B 223\&224 366

[5] Matsuda Y H, Inami T, Ohwada K, Murata Y, Nojiri H, Murakami Y, Ohta H, Zhang W, and Yoshimura Y 2007 J. Phys. Soc. Japan 76034702

[6] Jaccard D, Wilhelm H, Alami-Yadri K, and Vargoz E 1999 Physica B 259-261 1

[7] Holmes A T, Jaccard D, and Miyake K 2004 Phys. Rev. B 69024508

[8] Yuan H Q, Grosche F M, Deppe M, Geibel C, Sparn G, and Steglich F 2003 Science 3022104

[9] Miyake K, Narikiyo O, and Onishi Y 1999 Physica B 259-261 676

[10] Onishi Y and Miyake K 2000 J. Phys. Soc. Japan 693955

[11] Miyake K and Maebashi H 2001 J. Phys. Soc. Japan 711007

[12] Watanabe S, Imada M, and Miyake K 2006 J. Phys. Soc. Japan 75043710

[13] Monthoux P and Lonzarich G G 2004 Phys. Rev. B 69064517

[14] Fujiwara K, Hata Y, Kobayashi K, Miyoshi K, Takeuchi J, Shimaoka Y, Kotegawa H, Kobayashi T C, Geibel C, and Steglich F 2008 J. Phys. Soc. Japan 77123711

[15] Lengyel E, Nicklas C M, Jeevan H S, Sparn G, Geibel C, Steglich F, Yoshioka Y, and Miyake K 2009 Phys. Rev. B 80140513

[16] Miyake K 2007 J. Phys.: Condens Matter 19125201

[17] Watanabe S and Miyake K 2010 Phys. Status Solidi B 247490

[18] Watanabe S, Tsuruta A, Miyake K, and Flouquet 2009 J. Phys. Soc. Japan 78104706

[19] Saiga Y, Sugibayashi T, and Hirashima D 2008 J. Phys. Soc. Japan 77114710

[20] We refer to spatially-uniform and quantum-mechanically valence-fluctuating state with $n_{\mathrm{f}}<1$ as mixed valence. We refer to the state with larger $n_{\mathrm{f}}$ than that in the mixed-valence state at the FOVT and valence crossover as the Kondo state. Note that in the intermediate-coupling regime, $n_{\mathrm{f}}$ in the Kondo state is smaller than 1 [12, 18]. 
[21] Watanabe S and Miyake K 2010 J. Phys. Soc. Japan 79033707

[22] Watanabe S, Tsuruta A, Miyake K, and Flouquet J 2008 Phys. Rev. Lett. 100236401

[23] Petrovic C, Movshovich R, Jaime M, Pagliuso P G, Hundley M F, Sarrao J L, Fisk Z, and Thompson J D 2001 Europhys. Lett. $\mathbf{5 3} 354$

[24] Kawasaki S, Zheng G q, Kan H, Kitaoka Y, Shishido H, Ōnuki 2005 Phys. Rev. Lett. 94037007

[25] Takeuchi T, Inoue T, Sugiyama K, Aoki D, Tokiwa Y, Haga Y, Kindo K, and Ōnuki Y 2001 J. Phys. Soc. Japan 70877

[26] Kim J S, Alwood J, Kumar P, and Stewart G R 2002 Phys. Rev. B 65174520

[27] Palm E C, Murphy T P, Hall D, Tozer S W, Goodrich R G, and Sarrao J L 2003 Physica B 329-333 587

[28] Capan C, Bianchi A, Ronning F, Lacerda A, Thompson J D, Hundley M F, Pagliuso P G, Sarrao J L, and Movshovich R 2004 Phys. Rev. B 70180502

[29] Capan C, Balicas L, Murphy T P, Palm E C, Movshovich R, Hall D, Tozer S W, Hundley M F, Bauer E D, Thompson J D, Sarrao J L, DiTusa J F, Goodrich R G and Fisk Z 2009 Phys. Rev. B 80094518

[30] Watanabe S and Miyake K 2010 Phys. Rev. Lett. 105 186403; see also Watanabe S and Miyake K, preprint (arXiv:0906.3986)

[31] Haga Y, Inada Y, Harima H, Oikawa K, Murakawa M, Nakawaki H, Tokiwa Y, Aoki D, Shishido H, Ikeda S, Watanabe N, and Ōnuki Y 2001 Phys. Rev. B 6306503

[32] Shishido H, Settai R, Aoki D, Ikeda S, Nakawaki H, Nakamura N, Iisuka T, Inada Y, Sugiyama K, Takeuchi T, Kindo K, Kobayashi T C, Haga Y, Harima H, Aoki Y, Namiki T, Sato H, and Ōnuki Y 2002 J. Phys. Soc. Japan $\mathbf{7 1} 162$

[33] A. D. Christianson, E. D. Bauer, J. M. Lawrence, P. S. Riseborough, N. O. Moreno, P. G. Pagliuso, J. L. Sarrao, J. D. Thompson, E. A. Goremychkin, F. R. Trouw, M. P. Hehlen, and R. J. McQueeney 2004 Phys. Rev. B $\mathbf{7 0} 134505$

[34] Sidorov V A, Nicklas M, Pagliuso P G, Sarrao J L, Bang Y, Balatsky A V, and Thompson J D 2002 Phys. Rev. Lett. 89157004

[35] J. L. Sarrao, C. D. Immer, Z. Fisk, C. H. Booth, E. Figueroa, J. M. Lawrence, R. Modler, A. L. Cornelius, M. F. Hundley, G. H. Kwei, J. D. Thompson, and F. Bridges 1999 Phys. Rev. B 596855

[36] Koyama T, Matsumoto M, Tanaka T, Ishida H, Mito T, Wada S, and Sarrao J L 2002 Phys. Rev. B 66 014420

[37] Takeuchi T, Yasui S, Toda M, Matsushita M, Yoshiuchi S, Ohya M, Katayama K, Hirose Y, Yoshitani N, Honda F, Sugiyama K, Hagiwara M, Kindo K, Yamamoto E, Haga Y, Tanaka T, Kubo Y, Settai R, and Ōnuki Y 2010 J. Phys. Soc. Japan 79064609

[38] We note that each material has different strength of c-f hybridization $V$. Hence, the locus of the QCEP under the magnetic field depends on $V$ in Figs. 3(a) and 3(b), strictly speaking. To avoid confusion by drawing each locus (thick line with an arrow) for each material, we just drew one locus and schematic locations of $\mathrm{CeIrIn}_{5}, \mathrm{YbAgCu}_{4}$, and $\mathrm{YbAuCu}_{4}$, respectively, in Fig. 3(b).

[39] Honda F, Yasui S, Yoshiuchi S, Takeuchi T, Settai R, and Ōnuki Y 2010 J. Phys. Soc. Japan 79 in press

[40] Ohya M, Matsushita M, Yoshiuchi S, Takeuchi T, Honda F, Settai R, Tanaka T, Kubo Y, Onuki Y $2010 J$. Phys. Soc. Japan 79083601

[41] Hegger H, Petrovic C, Moshopoulou E G, Hundley M F, Sarrao J L, Fisk Z, and Thompson J D 2000 Phys. Rev. Lett. 84986

[42] Bao W, Pagliuso P G, Sarrao J L, Thompson J D, Fisk Z, Lynn J W, and Erwin R W 2003 Phys. Rev. B 67099903

[43] Knebel G, Aoki D, Brison J P, and Flouquet J 2008 J. Phys. Soc. Japan 77114704

[44] Park T, Sidorov V A, Ronning F, Zhu J-X, Tokiwa Y, Lee H, Bauer E D, Movshovich R, Sarrao J L, and Thompson J D 2008 Nature 456366

[45] Yashima M, Mukuda H, Kitaoka Y, Shishido H, Settai R, and Ōnuki Y 2009 Phys. Rev. B 79214528

[46] Shishido H, Settai R, Harima H, and Ōnuki Y 2005 J. Phys. Soc. Japan 741103

[47] Muramatsu T, Tateiwa N, Kobayashi T C, Shimizu K, Amaya K, Aoki D, Shishido H, Haga Y, and Ōnuki Y 2001 J. Phys. Soc. Japan 703362

[48] Park T and Thompson J D 2009 New Jpurnal of Physics 1105502

[49] A slight difference for the value of $P_{\mathrm{c}}$ exists in different samples: $P_{\mathrm{c}} \sim 1.75 \mathrm{GPa}$ in Ref. 44]

[50] Si Q, Rabello S, Ingersent K, and Smith J L 2001 Nature 413804

[51] Coleman P, Pépin C, Si Q, and Ramazashvili R 2001 J. Phys.: Condens. Matter 13 R723

[52] Kotliar G and Ruckenstein A E 1996 Phys. Rev. Lett. 571362

[53] Miyake K, Matsuura T, and Varma C M 1989 Solid State Commun. 711149

[54] Kuramoto Y and Miyake K 1990 J. Phys. Soc. Japan 592831

[55] Pham L D, Park T, Maquilon S, Thompson J D, and Fisk Z 2006 Phys. Rev. Lett. 97056404

[56] Yamamoto A, Wada S, and Sarrao J L 2007 J. Phys. Soc. Japan 76063709 
[57] Wada S, Yamamoto A, Ishida K, and Sarrao J L 2008 J. Phys.: Condens. Matter 20175201

[58] Gegenwart P, Westerkamp T, Krellner C, Tokiwa Y, Paschen S, Geibel C, Steglich F, Abrahams E, and Si Q 2007 Science 315969

[59] Gegenwart P, Steglich F, and Si Q, 2008 Nature Physics 4186 and references therein.

[60] Krellner C, Hartmann S, Pikul A, Oeschler N, Donath J G, Geibel C, Steglich F, and Wosnitza J 2009 Phys. Rev. Lett. 102196402

[61] Nakatsuji S, Kuga K, Machida Y, Tayama T, Sakakibara T, Karaki Y, Ishimoto H, Yonezawa S, Maeno Y, Pearson E, Lonzarich G G, Balicas L, Lee H, and Fisk Z 2008 Nature Physics 4603

[62] Okawa M, Matsunami M, Ishizaka K, Eguchi R, Taguchi M, Chainani A, Takata Y, Yabashi M, Tamasaku K, Nishino Y, Ishikawa T, Kuga K, Horie N, Nakatsuji S, and Shin S 2010 Phys. Rev. Lett. 104247201 